\title{
Effect of pore to throat size ratio on thermal dispersion in porous media
}

\author{
Turkuler Ozgumus, Moghtada Mobedi* \\ Department of Mechanical Engineering, Izmir Institute of Technology, Urla, Izmir 35430, Turkey
}

\section{A R T I C L E I N F O}

\section{Article history:}

Received 13 January 2015

Received in revised form

23 November 2015

Accepted 8 January 2016

Available online 6 February 2016

\section{Keywords:}

Porous media

Thermal dispersion

Volume averaging method

Throat effect

\begin{abstract}
A B S T R A C T
In this study, the effects of pore to throat size ratio on thermal dispersion of periodic porous media consisting of inline array of rectangular rods are investigated, numerically. The continuity, momentum and energy equations are solved for representative elementary volumes (REVs) of the porous media to obtain microscopic velocities in the voids between the rods and temperature distribution for entire of the REVs. Volume averaging method is employed to compute the macroscopic velocity and temperature values. There are velocity and temperature deviations between the macroscopic and microscopic values. These deviations are computed numerically and thermal dispersion coefficients of the porous media are determined. The aim of this study is to analyze the effects of pore to throat size ratio on the longitudinal and transverse thermal dispersion in the porous media. The study is performed for pore to throat size ratios between 1.63 and 7.46, porosities from 0.7 to 0.9 , and pore level Reynolds numbers between 1 and 100. It is found that in addition to the porosity and Reynolds number, the parameter of pore to throat size ratio plays an important role on thermal dispersion in a porous medium. It is found that there is an optimum value of pore to throat size ratio for maximum longitudinal thermal dispersion coefficient; however, the transverse thermal dispersion increases with the increasing of values of pore to throat size ratio.
\end{abstract}

๑) 2016 Elsevier Masson SAS. All rights reserved.

\section{Introduction}

A fluid can flow through the open pores that exist in a solid phase or between the solid particles. Flow in a porous medium is complex due to the discontinuity in flow field. The pore-level analysis of fluid flow and heat transfer for entire porous medium to determine velocity, pressure and temperature distributions is a difficult approach. To simplify the analysis, the macroscopic approach based on volume averaging technique was developed [1]. In the macroscopic approach, a porous medium consisting of different phases is considered as an imaginary continuum domain and the macroscopic governing equations are employed to determine the macroscopic velocity, pressure and temperature distributions for the entire porous medium. The macroscopic governing equations can be obtained by applying the volume averaging

\footnotetext{
* Corresponding author. Current address: Faculty of Engineering, Shizuoka University Hamamatsu Campus, 3-5-1 Johoku, Naka-ku, Hamamatsu, Japan.

E-mail addresses: turkulerozgumus@iyte.edu.tr (T. Ozgumus), moghtadamobedi@iyte.edu.tr, moghtada.mobedi@shizuoka.ac.jp (M. Mobedi).
}

technique on the corresponding microscopic governing equation. As a consequence of application, some extra terms (involving coefficients such as permeability, interfacial convective heat transfer coefficient and thermal dispersion coefficient) appear in the macroscopic momentum and energy equations. Thermal dispersion term in the macroscopic energy equation indicates the nonuniformities of the pore level velocity and temperature and as well the effect of hydrodynamic mixing. The effect of thermal dispersion on convection heat transfer in a porous medium becomes stronger as Reynolds number increases. Thermal dispersion also depends on other parameters such as Prandtl number, porosity, solid-to-fluid thermal conductivity ratio. Additionally, the shape of particles strongly influences thermal dispersion [2].

Thermal dispersion coefficient can be calculated experimentally or theoretically. The reported experimental studies on determination of thermal dispersion for packed beds filled with different particles were extensively reviewed and classified in Ref. [3]. Recent developments in computational technology lead researchers to determine thermal dispersion coefficient by using numerical methods. For instance, Sahraoui and Kaviany [4] considered two-dimensional periodic arrangement of cylinders 
and determined the longitudinal and transverse total thermal diffusivities, including both molecular diffusion and dispersion coefficients, by considering slip and no-slip temperature boundary conditions. It was shown that the ratio of total thermal diffusivity to thermal conductivity of the fluid changes with the square of Peclet number for inline arrangement while for the staggered arrangement the ratio increases linearly with Peclet number. It was also observed that for low Peclet numbers, the total thermal diffusivity is almost equal to the stagnant thermal conductivity of the porous medium and the thermal dispersion is negligible. Kuwahara et al. [5] studied periodical square rods in inline arrangement to determine transverse thermal dispersion and tortuosity. Two correlations for the determination of thermal dispersion, for high and low Peclet numbers, in terms of porosity, Peclet number and fluid thermal conductivity were suggested. Kuwahara and Nakayama [6] considered the same porous structure to find the longitudinal thermal dispersion and they found that the transverse thermal dispersion is substantially lower than longitudinal one. Correlations for the longitudinal thermal dispersion with porosity and Peclet number were proposed for the considered porous structure. Saada et al. [7] studied heat and fluid flow in porous media with the square rods and plus-shaped rods in inline and staggered arrangements. It was found that for the identical shape of rods, the longitudinal thermal dispersion is higher for the inline arrangement while the transverse one is higher for the staggered arrangement. Pedras and de Lemos [8] studied a periodic infinite porous medium consisting of elliptic rods. It was claimed that the type of thermal boundary condition has negligible effect on the longitudinal thermal dispersion value and only a slight effect on the transverse thermal dispersion could be observed. Xu et al. [9] studied periodic array of parallel plates and determined thermal dispersion analytically. It was shown that thermal dispersion can be influenced by the type of thermal conditions imposed on the medium since the imposed heat source condition affects the temperature non-uniformity in the channel. Alshare et al. [10] investigated the change of thermal dispersion in an array of square rods with inline arrangement by changing the representative elementary volume (REV) aspect ratio. Both transverse and longitudinal thermal dispersion conductivities were studied. It was found that the thermal dispersion depends on both Peclet number and flow angle, and the thermal dispersion coefficient in the flow direction is much larger than the thermal dispersion in the transverse direction to the flow. Jeong and Choi [11] investigated the longitudinal thermal dispersion in 2-D arrays of uniformly distributed circular and square cylinders, spheres and cubes by using lattice Boltzmann method. It was indicated that the inline arrangement causes higher longitudinal dispersion compared to the staggered arrangement and the dispersion depends on the arrangement rather than the shape of particles.
As seen from the above literature review, many studies on the determination of longitudinal and transverse thermal dispersion coefficients were reported. The effects of various parameters such as porosity, Reynolds and Prandtl numbers and fluid-to-solid thermal conductivity ratio on the thermal dispersion coefficient were investigated. However, to the best of our knowledge, no study on the effect of pore to throat size ratio on thermal dispersion has been reported. A porous medium can be described by different geometrical indicators such as porosity, nominal pore size and pore size distribution. Pore to throat size ratio is an important geometrical indicator of a porous medium and widely used to specify the structure of a porous medium. This indicator shows the degree of heterogeneity of the pores. As can be seen from Fig. 1, there are many types of porous media in which the effect of pore to throat size ratio should be taken into account. The contraction and expansion of fluid in a cell of metal foam (Fig. 1(a)) or cross cut pin fin array (Fig. 1(b)) for enhancing heat transfer are two examples for which the effect of pore to throat size ratio on heat and fluid flow should be taken into account. The effects of pore to throat size ratio on the pressure drop through the porous media and permeability are investigated prior to the present study [13]. The aim of the present study is to investigate the effect of pore to throat size ratio on the longitudinal and transverse thermal dispersion coefficients of porous media consisting of array of rectangular rods in inline arrangement, numerically. The velocity and temperature fields are obtained for the REV of the porous media and then the longitudinal and transverse thermal dispersion coefficients are calculated. The study shows that the thermal dispersion coefficients of two porous media with identical porosity, equivalent particle and hydraulic pore diameters but different pore to throat size ratios can be considerably different and the pore to throat size ratio plays an important role on the dispersion of heat in porous media. Hence, the involving of pore to throat size ratio as a structural indicator will provide more general correlations for macroscopic transport properties, which can be valid for various porous media.

\section{The considered porous media and computational domain}

The schematic of the considered porous media is shown in Fig. 2. The porous media is an infinite media consisting of rectangular rods in inline arrangement. Considering the periodicity of the porous structure, representative elementary volumes with the dimensions of $\mathrm{H} \times \mathrm{H}$ is chosen to investigate the pore to throat size ratio effect on the thermal dispersion coefficients of the porous media. The dimensions of REVs are constant for all studied cases in the present study. The rectangular particles have dimensions of $D_{x}$ and $D_{y}$ along $\mathrm{x}$ and $\mathrm{y}$ directions. The pore to throat size ratio is defined as $\beta=\mathrm{H} /$ $\left(H-D_{y}\right)$. The pore to throat size ratio and porosity are changed from 1.63 to 7.46 and from 0.7 to 0.9 , respectively. For instance,

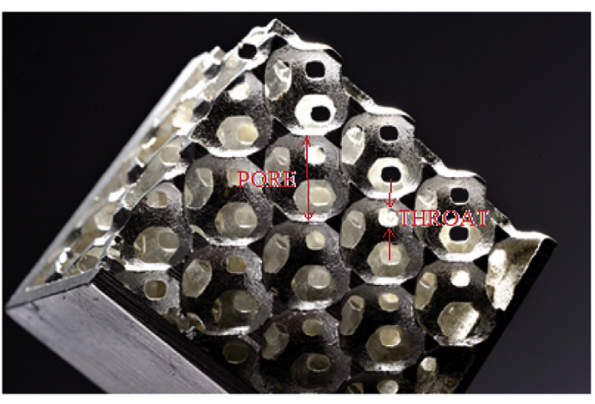

(a)
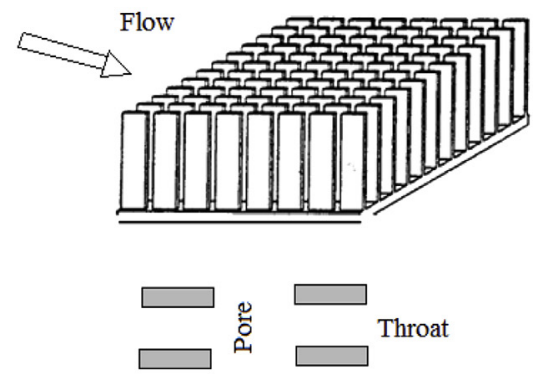

(b)

Fig. 1. Two periodic porous media in which the effect of pore to throat size ratio is significant, (a) Metal foam [12], (b) Cross Cut Pin Fins. 


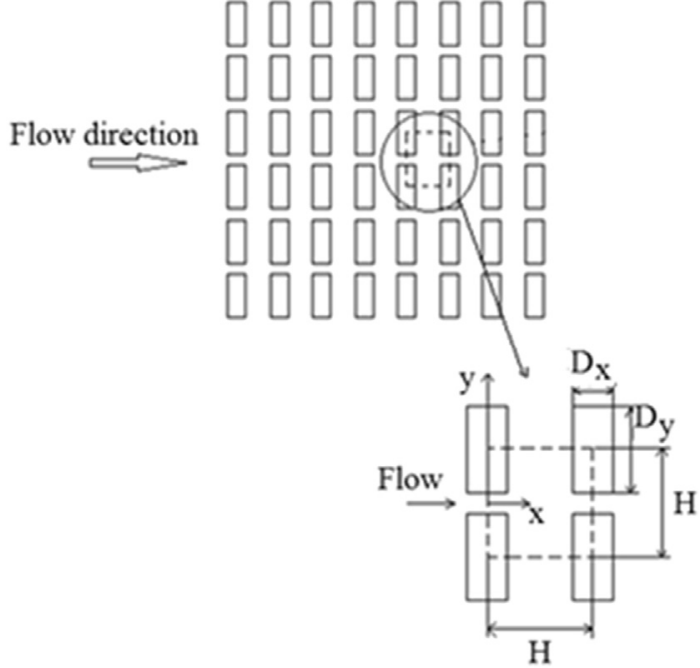

Fig. 2. The schematic view of the considered porous media.

Table 1 displays the geometrical parameters of the REVs with porosity of 0.7 for different values of $\beta$. As can be seen from the table, REVs with pore to throat size ratios of 1.63 and 4.44 have the same equivalent particle and pore hydraulic diameters when $\varepsilon=0.7$. The fluid flowing through the porous media is air with constant properties (density of $1.205 \mathrm{~kg} / \mathrm{m}^{3}$ and viscosity of $18.21 \cdot 10^{-6} \mathrm{~kg} / \mathrm{ms}$ ). The flow is laminar and macroscopically unidirectional. The pore level Reynolds number (i.e. $\operatorname{Re}=u H / v$ ) is changed between 1 and 100. The results are obtained for steady flow with hydrodynamically and thermally periodical and fullydeveloped conditions in REV. The present study is performed for the ratio of thermal conductivities of 2 .

\section{The microscopic and macroscopic governing equations}

The flow in the voids between the particles is assumed incompressible and steady. The fluid is a Newtonian fluid with constant thermophysical properties. The steady form of the continuity and momentum equations (Eqs. (1)-(3)) are solved to determine the velocity and pressure fields for the fluid flow in the voids between the rods. After obtaining the velocity field in the porous media, the energy equations for solid and fluid phases (Eqs. (4) and (5)) are solved to obtain the temperature distributions in the REVs.

$$
\frac{\partial u}{\partial x}+\frac{\partial v}{\partial y}=0
$$

$\rho_{f}\left(u \frac{\partial u}{\partial x}+v \frac{\partial u}{\partial y}\right)=-\frac{\mathrm{d} p}{\mathrm{~d} x}+\mu\left(\frac{\partial^{2} u}{\partial x^{2}}+\frac{\partial^{2} u}{\partial y^{2}}\right)$

Table 1

A sample of geometrical parameters of the investigated REVs.

\begin{tabular}{llllll}
\hline & $\beta=1.63$ & $\beta=2.21$ & $\beta=3.04$ & $\beta=4.44$ & $\beta=7.46$ \\
\hline $\mathrm{A}^{*}$ & 0.5 & 1 & 1.5 & 2 & 2.5 \\
$\varepsilon$ & 0.7 & 0.7 & 0.7 & 0.7 & 0.7 \\
$\mathrm{D}_{\mathrm{x}} / \mathrm{H}$ & 0.776 & 0.547 & 0.446 & 0.388 & 0.346 \\
$\mathrm{D}_{\mathrm{y}} / \mathrm{H}$ & 0.388 & 0.547 & 0.670 & 0.776 & 0.866 \\
$\mathrm{~d}_{\mathrm{h}} / \mathrm{H}$ & 1.207 & 1.276 & 1.250 & 1.207 & 1.155 \\
$\mathrm{~d}_{\mathrm{p}} / \mathrm{H}$ & 0.776 & 0.820 & 0.804 & 0.776 & 0.742 \\
\hline
\end{tabular}

$\rho_{f}\left(u \frac{\partial v}{\partial x}+v \frac{\partial v}{\partial y}\right)=-\frac{\mathrm{d} p}{\mathrm{~d} y}+\mu\left(\frac{\partial^{2} v}{\partial x^{2}}+\frac{\partial^{2} v}{\partial y^{2}}\right)$

$\rho_{f} c_{p f}\left(\mathrm{u} \frac{\partial \mathrm{T}_{\mathrm{f}}}{\partial \mathrm{x}}+\mathrm{v} \frac{\partial \mathrm{T}_{\mathrm{f}}}{\partial \mathrm{y}}\right)=k_{f}\left(\frac{\partial^{2} \mathrm{~T}_{\mathrm{f}}}{\partial \mathrm{x}^{2}}+\frac{\partial^{2} \mathrm{~T}_{\mathrm{f}}}{\partial \mathrm{y}^{2}}\right)$

$\frac{\partial^{2} \mathrm{~T}_{s}}{\partial \mathrm{x}^{2}}+\frac{\partial^{2} \mathrm{~T}_{s}}{\partial \mathrm{y}^{2}}=0$

where $\mathrm{u}$ and $\mathrm{v}$ are the velocity components along $\mathrm{x}$ and $\mathrm{y}$ directions, and $\mathrm{p}$ is pressure. $\mathrm{T}_{\mathrm{f}}$ and $\mathrm{T}_{\mathrm{s}}$ represent the fluid and solid temperatures, respectively.

The continuity, momentum and energy equations given by Eqs. (1)-(5) are microscopic equations for the pore level analysis of the porous media. The macroscopic equations for the fluid flow and heat transfer can be derived from the microscopic equations by using the volume averaging method [1]. The volume average of a dependent quantity over a REV can be defined by Eqs. (6) and (7). Eq. (6) shows the total volume average of a quantity $\phi$ while Eq. (7) is used to find the intrinsic volume averaged value of $\phi$ for any of phases in a porous medium.

$\langle\phi\rangle=\frac{1}{V} \int_{V} \phi \mathrm{d} V$

$\langle\phi\rangle^{X}=\frac{1}{V_{x}} \int_{V_{x}} \phi \mathrm{d} V$

where $\mathrm{V}$ is the total volume of the REV and $\mathrm{V}_{\mathrm{x}}$ is the volume of the considered phase in the REV. The variable $\phi$ can be velocity or temperature in the present study. The deviation of the microscopic dependent quantity from the intrinsic macroscopic value is defined by Eq. (8):

$\phi^{\prime}=\phi-\langle\phi\rangle^{f}$

Taking volume integral of the energy equations (Eqs. (4) and (5)) and employing Eqs. (6)-(8) and finally rearranging the terms with proper integration rules yield the macroscopic energy equations of the fluid and solid phases [3]:

$$
\begin{aligned}
\left(\rho \mathrm{c}_{\mathrm{p}}\right)_{\mathrm{f}}\langle\overrightarrow{\mathrm{u}}\rangle \cdot\langle\mathrm{T}\rangle^{\mathrm{f}}= & \mathrm{k}_{\mathrm{f}} \varepsilon \nabla^{2}\langle\mathrm{~T}\rangle^{\mathrm{f}}+\nabla\left(\frac{1}{\mathrm{~V}} \int_{\mathrm{A}_{\mathrm{sf}}} \mathrm{k}_{\mathrm{f}} \mathrm{T} \mathrm{dA}\right)+\frac{1}{\mathrm{~V}} \int_{\mathrm{A}_{\mathrm{sf}}} \mathrm{k}_{\mathrm{f}} \nabla \mathrm{T} \mathrm{dA} \\
& -\left(\rho \mathrm{c}_{\mathrm{p}}\right)_{\mathrm{f}} \nabla \cdot\left\langle\mathrm{T}^{\prime} \overrightarrow{\mathrm{u}}^{\prime}\right\rangle
\end{aligned}
$$

$\mathrm{k}_{S}(1-\varepsilon) \nabla^{2}\langle\mathrm{~T}\rangle^{S}-\nabla\left(\frac{1}{\mathrm{~V}} \int_{\mathrm{A}_{\mathrm{sf}}} \mathrm{k}_{\mathrm{s}} \mathrm{TdA}\right)-\frac{1}{\mathrm{~V}} \int_{\mathrm{A}_{\mathrm{sf}}} \mathrm{k}_{\mathrm{s}} \nabla \mathrm{T} \mathrm{dA}=0$

The first terms on the right hand side of Eq. (9) and left hand side of Eq. (10) are the thermal diffusion terms which represent the diffusion heat transfer in the fluid and solid phases. Similarly, the second terms relate to the thermal tortuosity $[2,14]$. The thermal tortuosity term regards the change of the thermal diffusion path due to the different thermal conductivities of solid and fluid [3]. The third terms show the heat transfer between the solid phase and the fluid flowing in the voids and these terms in Eqs. (9) and (10) should be equal to each other. The last term in the macroscopic energy equation of the fluid phase (Eq. (9)) is named as thermal 
dispersion. It is caused by the hydrodynamic mixing effects and the non-uniformities of the pore level velocity and temperature fields [3]. If the thermal equilibrium between two phases is valid, the macroscopic energy equations can be combined into a single macroscopic energy equation for the porous medium by setting $\langle T\rangle=\langle T\rangle^{f}=\langle T\rangle^{S}$

$$
\begin{aligned}
\left(\rho \mathrm{c}_{\mathrm{p}}\right)_{\mathrm{f}}\langle\overrightarrow{\mathrm{u}}\rangle \cdot\langle T\rangle= & \mathrm{k}_{\mathrm{e}} \nabla^{2}\langle T\rangle-\nabla\left(\frac{1}{\mathrm{~V}} \int_{\mathrm{A}_{\mathrm{sf}}}\left(k_{\mathrm{s}}-\mathrm{k}_{\mathrm{f}}\right) \mathrm{TdA}\right) \\
& -\left(\rho \mathrm{c}_{\mathrm{p}}\right)_{\mathrm{f}} \nabla \cdot\left\langle\mathrm{T}^{\prime} \overrightarrow{\mathrm{u}}^{\prime}\right\rangle
\end{aligned}
$$

where $\mathrm{k}_{\mathrm{e}}$ is the equivalent (stagnant) thermal conductivity and can be found by using Eq. (12).

$k_{e}=k_{S}(1-\varepsilon)+k_{f} \varepsilon$

Both the thermal tortuosity and thermal dispersion terms can be written in terms of diffusion heat transport. $\rho_{f} c_{p f}\left\langle\vec{u}^{\prime} T^{\prime}\right\rangle$ term is analogous with turbulent heat flux in turbulent convection heat transfer, which accounts for the contributions from the mechanical dispersion. Hence, the thermal dispersion term can be modelled by a gradient-type diffusion hypothesis and written as below [14]:

$-\rho_{f} c_{p f} \nabla \cdot\left\langle T^{\prime} \vec{u}^{\prime}\right\rangle=k_{\text {dis }} \nabla^{2}\langle T\rangle$

Thermal dispersion is a tensor quantity and has different components depending on the dimension of heat transfer problem. For a two dimensional heat transfer problem in porous media, the dispersion heat flux components can be shown as below by using thermal dispersion tensor:

$\left[\begin{array}{l}q_{d i s, X}^{\prime \prime} \\ q_{d i s, y}^{\prime \prime}\end{array}\right]=\left[\begin{array}{ll}k_{d i s, X X} & k_{d i s, X Y} \\ k_{d i s, Y X} & k_{d i s, Y Y}\end{array}\right]\left[\begin{array}{l}-\partial T / \partial x \\ -\partial T / \partial y\end{array}\right]$

where $q_{d i s, x}^{\prime \prime}$ and $q_{d i s, y}^{\prime \prime}$ are heat fluxes due to thermal dispersion in the longitudinal and transverse directions. $k_{d i s, X X}, k_{d i s, X Y}, k_{d i s, Y X}$ and $k_{d i s, Y Y}$ are the components of the thermal dispersion tensor in different directions. For the present problem, the porous media structure is periodical in $\mathrm{x}$ and $\mathrm{y}$ directions; hence, the values of $k_{d i s, X Y}$ and $k_{d i s, Y X}$ are zero. However the components of $k_{d i s, X X}$ and $k_{d i s, Y Y}$ exist and their values are different from each other. Eq. (11) can be rewritten as below by using the thermal dispersion and thermal tortuosity coefficients.

$\left(\rho \mathrm{c}_{\mathrm{p}}\right)_{\mathrm{f}}\langle\overrightarrow{\mathrm{u}}\rangle \cdot\langle T\rangle=\mathrm{k}_{\mathrm{e}} \nabla^{2}\langle T\rangle+k_{\text {tor }} \nabla^{2}\langle T\rangle+k_{\text {dis }} \nabla^{2}\langle T\rangle$
Since the values of thermal conductivity of solid and fluid phases are close to each other $\left(\mathrm{k}_{\mathrm{s}} / \mathrm{k}_{\mathrm{f}}=2\right)$, the term of thermal tortuosity can be neglected [15]. Then, the RHS of Eq. (15) can be written as below by defining the effective thermal conductivity:

$\left(\rho c_{\mathrm{p}}\right)_{\mathrm{f}}\langle\overrightarrow{\mathrm{u}}\rangle \cdot\langle T\rangle=\mathrm{k}_{\mathrm{eff}} \nabla^{2}\langle T\rangle$

$k_{\text {eff }}=\mathrm{k}_{\mathrm{e}}+k_{\text {dis }}$

To determine the thermal dispersion coefficients in the longitudinal and transverse directions, the equality given in Eq. (13) can be used with the assumption of linear temperature gradient in the corresponding direction of the REV. Eqs. (18) and (19) can be employed to find the thermal dispersion coefficients in $\mathrm{x}$ (longitudinal) and y (transverse) directions.

$k_{d i s, X X}=-\frac{1}{\Delta T / \Delta x} \frac{\rho_{f} c_{p f}}{V} \int_{V}\left(T_{f}-\left\langle T_{f}\right\rangle^{f}\right)\left(u-\langle u\rangle^{f}\right) \mathrm{d} V$
$k_{d i s, Y Y}=-\frac{1}{\Delta T / \Delta y} \frac{\rho_{f} c_{p f}}{V} \int_{V}\left(T_{f}-\left\langle T_{f}\right\rangle^{f}\right)\left(v-\langle v\rangle^{f}\right) \mathrm{d} V$

In order to determine the longitudinal and transverse thermal dispersion coefficients by employing Eqs. (18) and (19) both the microscopic and macroscopic velocity and temperature values should be known. The boundary conditions for the microscopic flow and energy equations (Eqs. (1)-(5)) for the REVs are shown in Table 2. As seen from the table, no-slip temperature and continuous heat flux boundary conditions are applied to the solid-fluid interface. At the fluid outlet boundary, no change of the velocity and temperature gradients is set, which means no diffusive transport exists at this boundary. At the fluid inlet boundary, a velocity profile $f(y)$ which fulfils the requirement of periodicity of flow is provided for the velocity in main flow direction and the velocity component in transverse direction is assumed zero.

For the computation of the longitudinal thermal dispersion coefficient, no change of the velocity components and the temperature in the y direction are chosen as boundary conditions for the top and bottom boundaries. For the longitudinal thermal dispersion, the temperature values at the solid boundaries of the inlet and outlet of the REVs are set as $\mathrm{T}_{\text {ref }}$ and $\mathrm{T}_{\text {ref }}+\Delta \mathrm{T}$, respectively. Here, $T_{\text {ref }}$ is a chosen reference temperature and $\Delta \mathrm{T}$ is the imposed macroscopic temperature difference value employed in Eq. (18). For the fluid inlet boundary, a temperature profile $\mathrm{g}(\mathrm{y})$, which is obtained by using an iterative procedure, is applied as temperature boundary condition. For the transverse thermal dispersion

Table 2

\begin{tabular}{|c|c|c|}
\hline Boundary & For longitudinal thermal dispersion & For transverse thermal dispersion \\
\hline At on the solid-fluid interfaces & $T_{f}=T_{s}, \vec{n}_{f s} \cdot\left(k_{f} \nabla T_{f}\right)=\vec{n}_{f s} \cdot\left(k_{s} \nabla T_{s}\right)$ & \\
\hline At the fluid outlet boundary & $\frac{\partial u}{\partial x}=v=\frac{\partial T}{\partial x}=0$ & \\
\hline At the fluid inlet boundary & $u=f(y), v=0$ & \\
\hline & $T_{f}=g(y)$ & $T_{f}=h(y)$ \\
\hline At the top and bottom boundaries & $\frac{\partial \vec{u}}{\partial y}=\frac{\partial T}{\partial y}=0$ & $\begin{array}{l}T(y=-H / 2)=T_{r e f} \\
T(y=H / 2)=T_{r e f}+\Delta T\end{array}$ \\
\hline At the solid boundaries of inlet and outlet rods & $\begin{array}{l}T_{s}(x=0)=T_{r e f} \\
T_{s}(x=H)=T_{r e f}+\Delta T\end{array}$ & $T_{s}(x=0)=T_{s}(x=H)=h(y)$ \\
\hline
\end{tabular}

Boundary conditions imposed to the REVs for the determination of thermal dispersion coefficients.

\section{Notes:}

$\mathrm{T}_{\text {ref }}$ is the reference temperature value.

$\vec{n}_{f s}$ shows normal direction at the interface.

$\Delta \mathrm{T}$ is the imposed macroscopic linear temperature difference.

$\mathrm{f}(\mathrm{y})$ is the velocity profile.

$\mathrm{g}(\mathrm{y})$ and $\mathrm{h}(\mathrm{y})$ are the temperature profiles. 
coefficient, the desired macroscopic temperature difference should exist in the transverse direction. That's why the uniform temperature as $\mathrm{T}_{\text {ref }}$ and $\mathrm{T}_{\text {ref }}+\Delta \mathrm{T}$ are applied to the bottom and top boundaries, respectively, and a macroscopic temperature gradient in the transverse direction is generated. For the solid boundaries at the inlet and outlet of the REV and the fluid inlet boundary, a linear temperature profile $\mathrm{h}(\mathrm{y})$ is set. The iterative procedure for finding of $\mathrm{g}(\mathrm{y})$ and $\mathrm{h}(\mathrm{y})$ functions is explained in the next section.

\section{Numerical procedure and computational details}

The microscopic fluid flow and energy equations are solved for the considered REVs, computationally. The grid independency studies are performed with different grid sizes and the variation of thermal dispersion with number of grids were checked. A sample of grid independency for porous media with $\beta=7.46$ and $\beta=1.63$ when $\operatorname{Re}=100$ and $\varepsilon=0.9$ is shown in Fig. 3. As can be seen, the grid size of $500 \times 250$ for the half of the REV is appropriate. A commercial code based on the finite volume method is used to solve the governing equations (ANSYS 12). The power law scheme is employed for the discretization of the convection terms in the momentum and energy equations. SIMPLE method is used for handling the pressure-velocity coupling. The residual convergence criteria are set to $10^{-9}$ for fluid motion equations and $10^{-12}$ for energy equation.

To provide periodic boundary conditions for the REVs and determine $\mathrm{f}(\mathrm{y})$ and $\mathrm{g}(\mathrm{y})$ functions, iterative methods are used. Firstly, the flow inlet and outlet boundaries are made periodical by solving the continuity and momentum equations by using the obtained outlet velocity profile as the inlet boundary condition for the next iterative computation step. The procedure is terminated when the velocity profiles at the inlet and outlet of the REVs become identical. Additionally, the permeability value is found for each iterative run and the convergence of the permeability to a constant value is also checked. After obtaining periodical flow condition in the REV, the energy equation is solved iteratively to provide macroscopically linear temperature gradient along the desired direction.

For the calculation of the longitudinal thermal dispersion, as it was mentioned before, the temperature values at the solid boundaries of the inlet and outlet of the REV are set as $\mathrm{T}_{\text {ref }}$ and $\mathrm{T}_{\text {ref }}+\Delta \mathrm{T}$, respectively. In order to provide the same $\Delta \mathrm{T}$ for the fluid inlet and outlet boundaries, an iterative procedure is employed. A uniform fluid temperature for the inlet boundary is selected and the temperature profile at the outlet boundary is obtained. The macroscopic temperature difference $(\Delta \mathrm{T})$ is subtracted from the obtained outlet fluid temperature profile and then used as the inlet

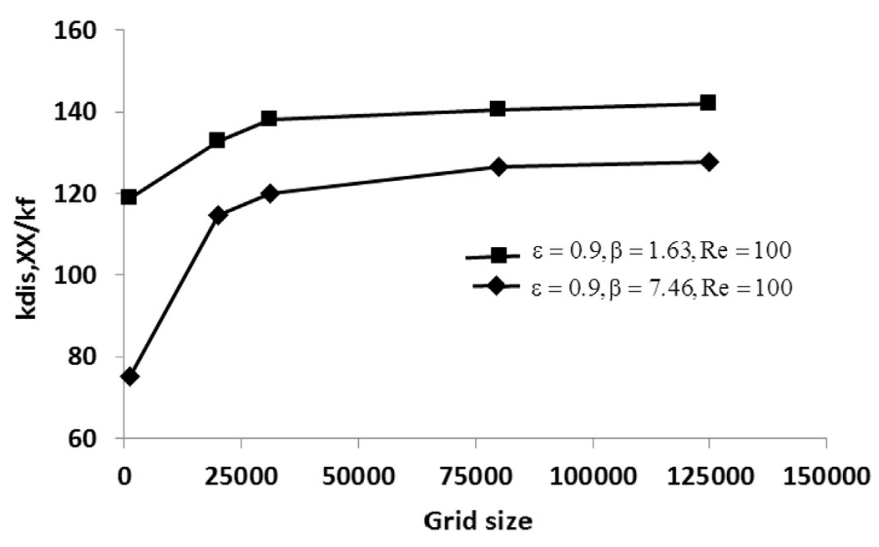

Fig. 3. Two samples of grid independency study.

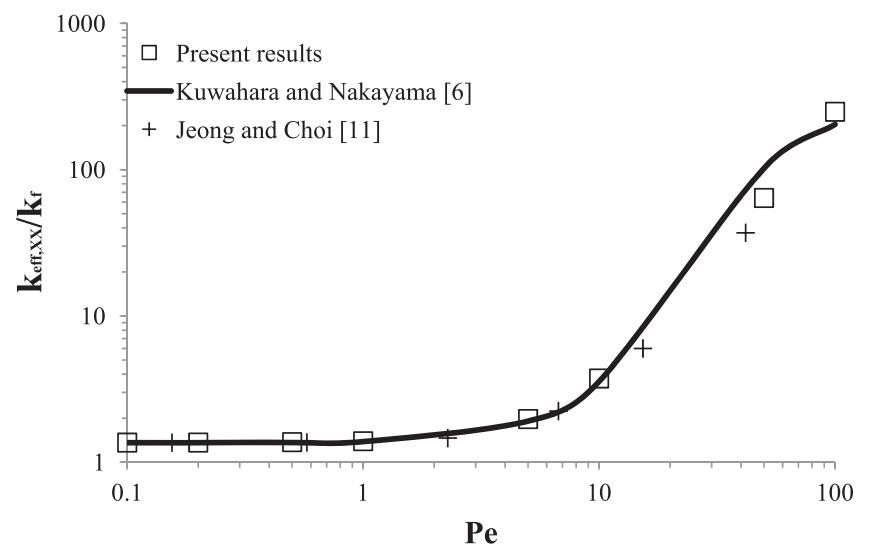

(a)

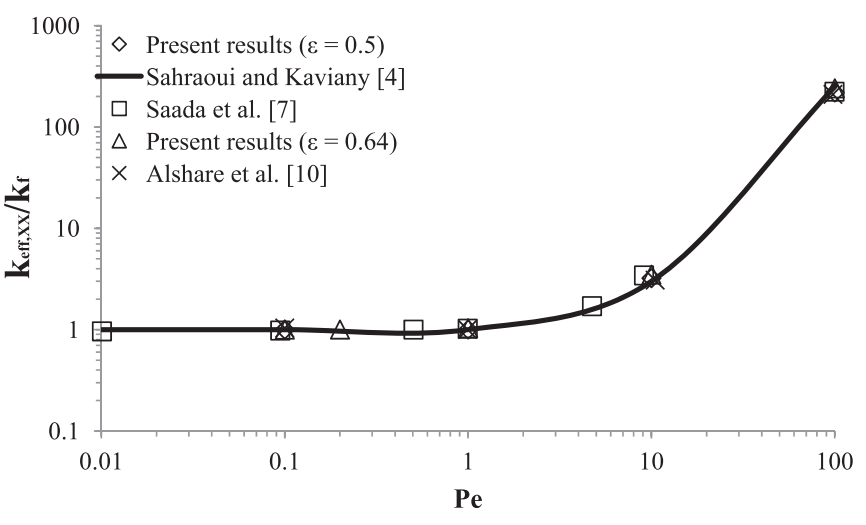

(b)

Fig. 4. The comparison of the present results obtained for the longitudinal effective thermal conductivity with the results of reported studies for (a) $\varepsilon=0.64$ and $k_{s} / k_{f}=2$, (b) $\varepsilon=0.64$ and $0.5, \mathrm{k}_{\mathrm{s}} / \mathrm{k}_{\mathrm{f}}=1$.

boundary condition. The procedure continues until the following condition is satisfied:

$T(H, y)-T(0, y)=\Delta T$

The inlet temperature profile which satisfies Eq. (20) is shown by $g(y)$. The temperature profile of $g(y)$ fulfils the macroscopic

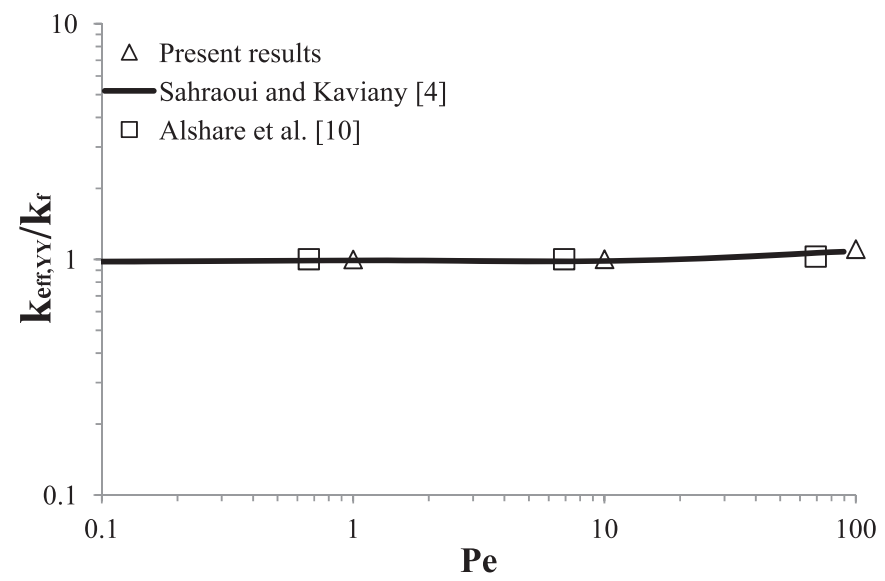

Fig. 5. The comparison of the present results obtained for the transverse effective thermal conductivity with the results of reported studies for $\varepsilon=0.75$ and $\mathrm{k}_{\mathrm{s}} / \mathrm{k}_{\mathrm{f}}=1$. 
temperature periodicity of REV and additionally helps to create a macroscopic linear temperature gradient along the flow direction.

For the calculation of the transverse thermal dispersion, the same periodic velocity distribution is used. As it was mentioned before, the desired temperature difference should be in the transverse direction. That is why; a constant and uniform temperature are applied to the bottom and top boundaries (including symmetry and solid boundaries) as $\mathrm{T}_{\text {ref }}$ and $\mathrm{T}_{\text {ref }}+\Delta \mathrm{T}$, respectively. A linear temperature profile $\mathrm{h}(\mathrm{y})$, which changes from $\mathrm{T}_{\text {ref }}$ to $\mathrm{T}_{\mathrm{ref}}+\Delta \mathrm{T}$ is provided to the inlet boundary including both solid and fluid boundaries. The same profile is also set to the outlet solid boundary. Hence, the macroscopically linear temperature gradient (including fluid region) is generated along the transverse direction of flow.

\section{Results and discussion}

The validation of the present numerical study is performed by the comparison of the obtained numerical results with the reported results in the literature for porous media consisting of square rods in inline arrangement. The comparisons of obtained effective thermal conductivity ratio $\left(\mathrm{k}_{\mathrm{eff}} / \mathrm{k}_{\mathrm{f}}\right)$ in the longitudinal direction with the reported results are shown in Fig. 4 for different values of porosity and solid-to-fluid thermal conductivity ratios. Furthermore, the variation of the effective thermal conductivity ratio in the transverse direction with $\mathrm{Pe}$ and its comparison with the available studies in the literature are displayed in Fig. 5. The obtained results of the present study are in good agreement with those values reported in the literature for both longitudinal and transverse thermal dispersion.

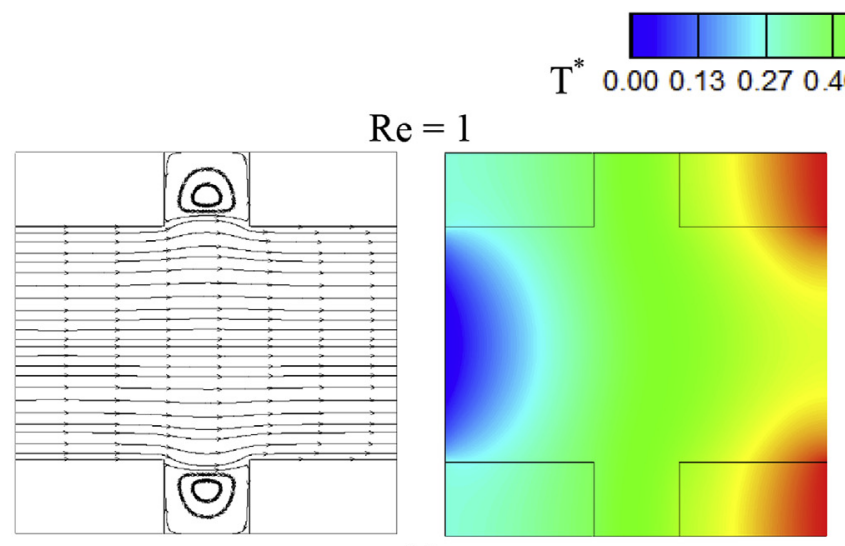

(a)

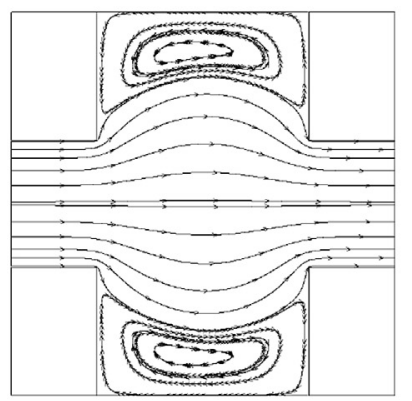

(c)
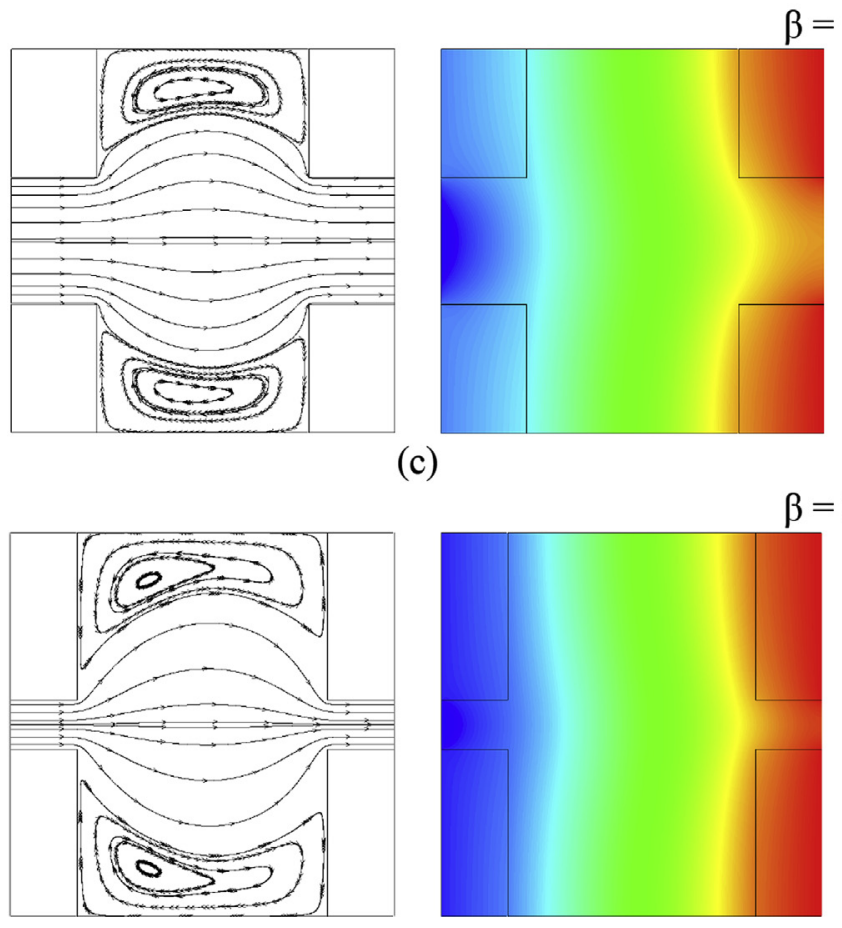

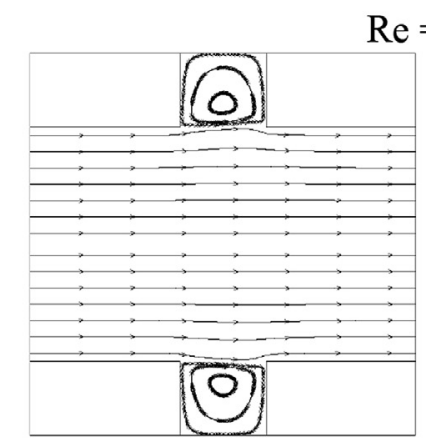

(b)
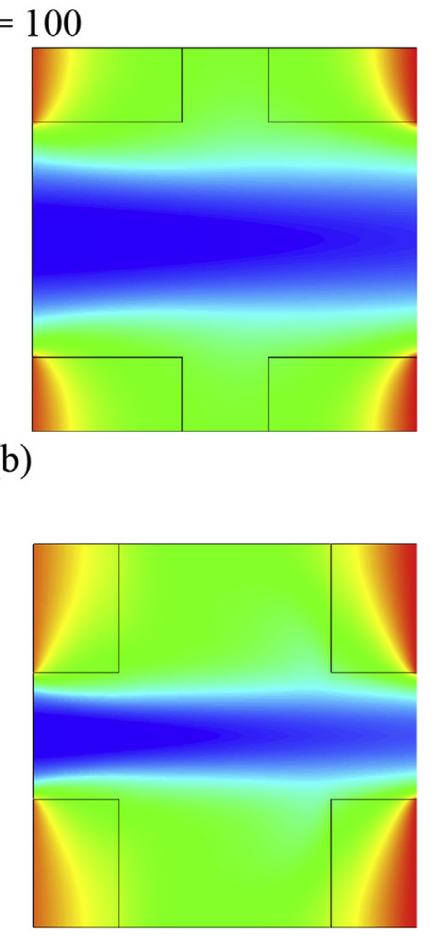

(d)
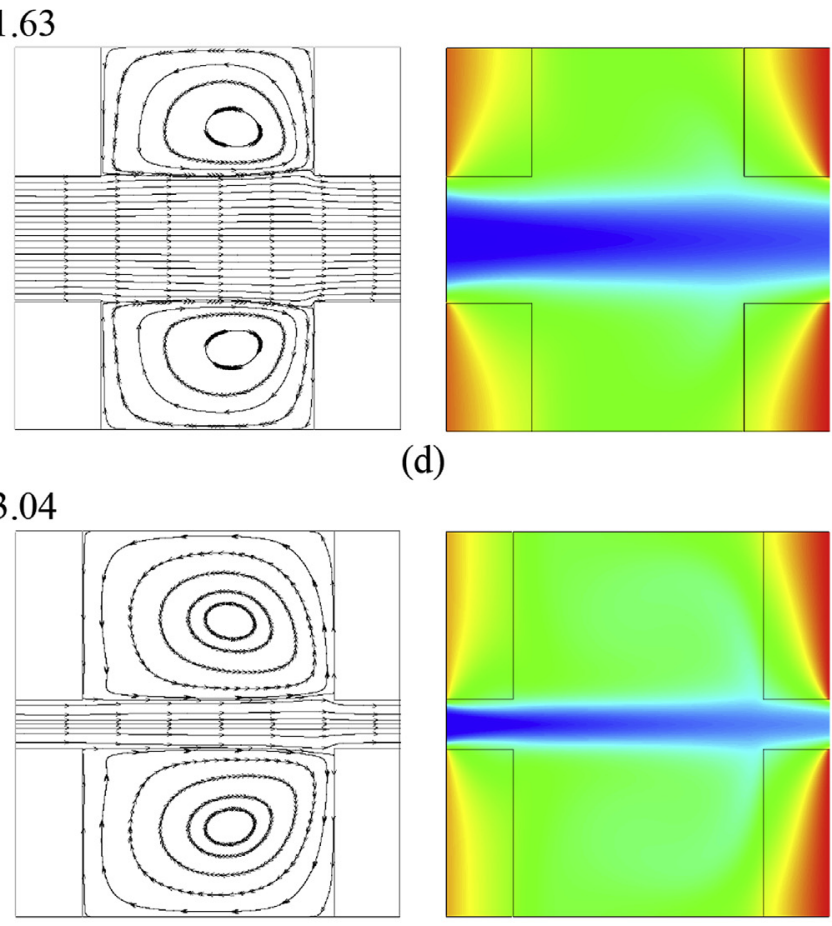

(e)

$$
\beta=7.46
$$

Fig. 6. The streamlines and dimensional temperature contours with longitudinal temperature gradient for porous media with porosity of 0.7 . 
\begin{tabular}{|l|l|l|l|l|l|l|}
\hline & & & & & & \\
\hline
\end{tabular}

$\operatorname{Re}=1$

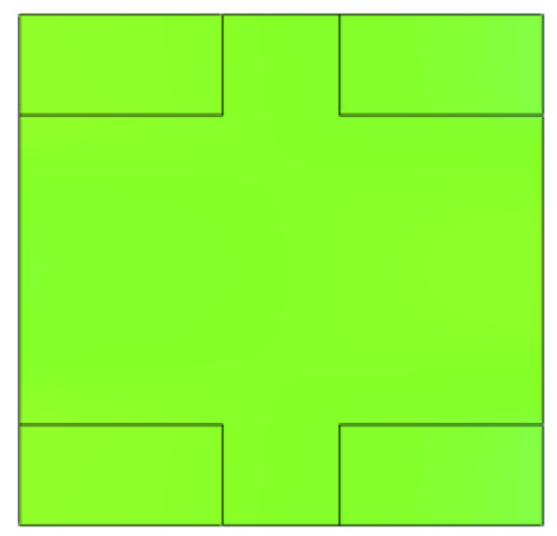

(a)

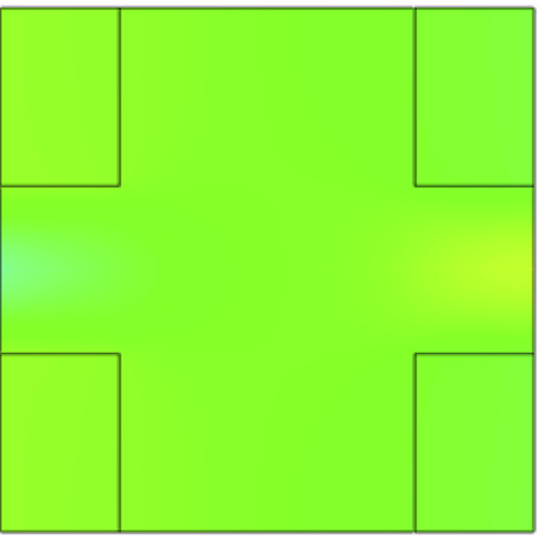

(c)

$\beta=3.04$

$$
\beta=1.63
$$
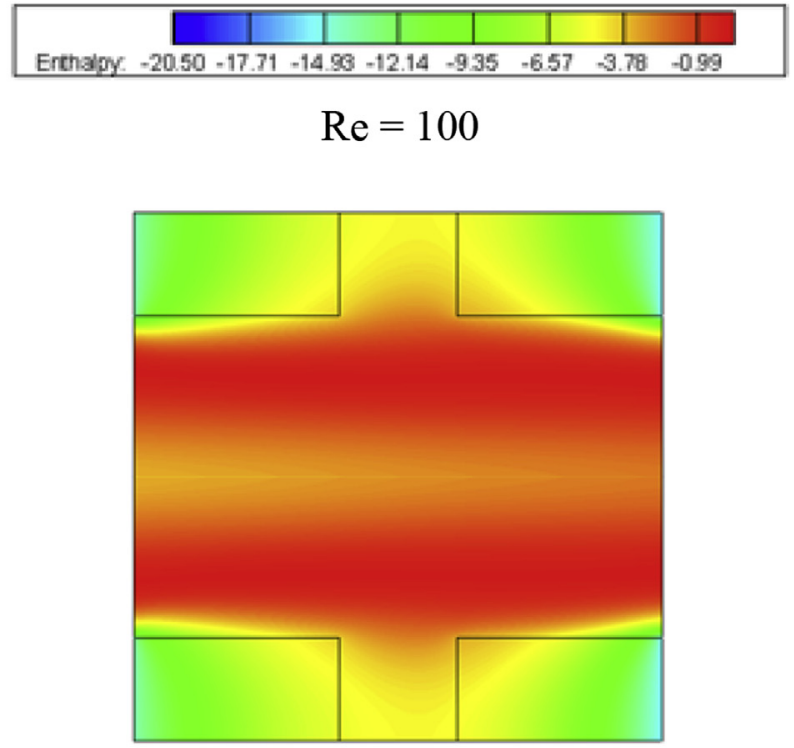

(b)

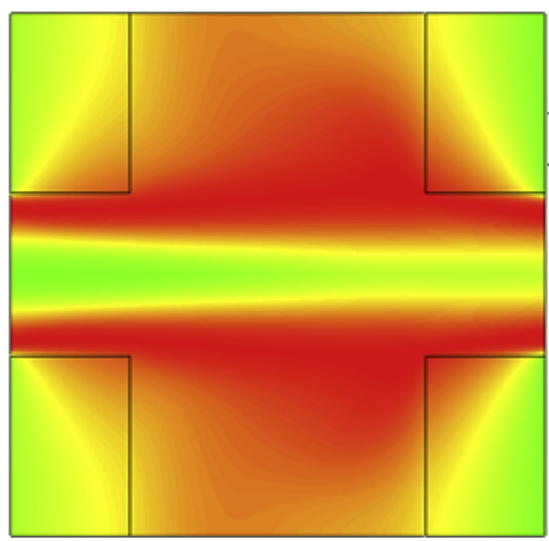

(d)

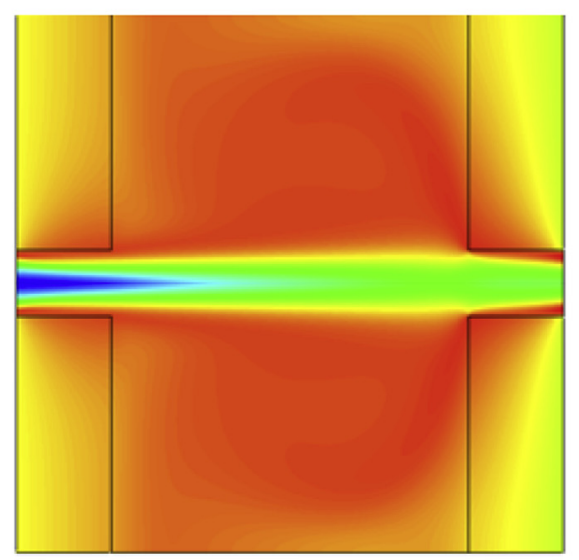

(f)

(e)

$$
\beta=7.46
$$

Fig. 7. The distributions of $\left(u^{\prime} T^{\prime}\right)^{*}$ in the REVs for porous media with $\varepsilon=0.7$. 
By solving the microscopic equations (Eqs. (1)-(5)) with using the aforementioned boundary conditions, the pore-level velocity and temperature distributions inside the REVs are obtained. In order to use the same legend for the presented results, the dimensionless temperature and velocity values are defined as:

$T^{*}=\frac{T-T_{\min }}{T_{\max }-T_{\min }}$

$u^{*}=\frac{u}{\langle u\rangle}$

where $T_{\min }$ and $T_{\max }$ are the minimum and maximum temperature values in the REVs. Additionally, a dimensionless value for $u^{\prime} T^{\prime}$ is defined in order to explain the variation of longitudinal thermal dispersion with pore to throat size ratio.

$\left(u^{\prime} T^{\prime}\right)^{*}=\frac{u^{\prime} T^{\prime}}{\langle u\rangle \Delta T}$

where $\Delta \mathrm{T}$ is macroscopic temperature difference.

In Fig. 6, the streamlines and temperature contours in REVs with porosity of 0.7 , pore to throat size ratios of $1.63,3.04$ and 7.46 are presented for Reynolds numbers of 1 and 100 . As it was mentioned before, the temperature contours show the distribution of dimensionless temperature (i.e., $T^{*}$ ) in the REVs. Fig. 6(a) shows the streamlines and dimensionless temperature distributions for the pore to throat size ratio of 1.63 , porosity of 0.7 and $R e=1$. As can be seen from the streamline patterns, the main flow along the REV goes straight like a channel flow since the particles (i.e. rods) are almost flat in flow direction. A small flow penetration to the gaps between the rods can be observed. There are secondary flows in the upper and lower gaps between particles. Temperature almost linearly drops from the left to the right side of the REV. Fig. 6(b) presents the streamlines and dimensionless temperature contours for the same REV when $R e=100$. The streamlines in the REV do not change very much, however a strong convection effect in the flow direction can be observed from the isotherms. The streamlines and dimensionless temperature distribution in the porous medium with the same porosity but pore to throat size ratio of 3.04 when $\operatorname{Re}=1$ are shown in Fig. 6(c). The main flow penetrates to the upper and lower gaps and compresses the secondary flows in the gaps. The dimensionless temperature distributions in Fig. 6(a) and (c) are similar; however, the linearity of the temperature distributions in the flow direction can be observed more clearly in Fig. 6(c). By increasing Re from 1 to 100 for the REV shown in Fig. 6(c), the streamlines and dimensionless temperature distribution considerably change as can be seen from Fig. 6(d). The main flow resembles the channel flow and the secondary flows cover the entire gaps. Similar to Fig. 6(b), a strong convection effect can be seen in the centre of the REV due to high value of Re. Furthermore, the collision of the main flow onto the vertical walls of the particles at the right side of the REV causes the entrance of cold fluid to the gaps between the particles and decreases the temperature inside the gaps. The streamlines and dimensionless temperature contour in the REV with $\beta=7.46$ for the flow with $\mathrm{Re}=1$ are shown in Fig. 6(e). The penetration of the main flow into the upper and lower gaps becomes stronger, the shapes of the secondary flows are distorted and the centres of the secondary flows move nearer to the inlet section. The linear temperature gradient along the longitudinal direction is obvious from the temperature distribution. The streamlines and temperature contours are presented in Fig. 6(f) for $R e=100$ of the same REV. Because of high Re flow, the main flow has nearly straight streamlines through the REV. Similar to the flow in Fig. 6(d), the collision of the main flow to the solid particles at the outlet of the REV causes the entrance of fluid particles to the upper and lower gaps. The cooling effect in the gaps is more obvious in the temperature contour of Fig. 6(f). For the all presented figures, the linearity of temperature gradient is observed more clearly for low Re because of the low velocities and convection effect. Thermal dispersion is resulted due to fluctuations between actual temperature and velocity and averaged ones in pores of porous media. As can be seen from Fig. 6, the aforementioned fluctuations of temperature is very low for $\operatorname{Re}=1$ and furthermore the magnitude of velocity fluctuations is also small because of low velocity values. Hence a negligible thermal dispersion can be expected for the flows with low pore level Reynolds numbers. As the value of Re increases, the temperature non-uniformities in the REVs increase and consequently the temperature fluctuations also increase. The magnitudes of the velocity fluctuations increase due to the increase of pore level velocity. That's why the increase of longitudinal thermal dispersion with Reynolds number can be expected.

The change of thermal dispersion with pore to throat size ratio and Reynolds number can be understood better by examining the distribution of dimensionless $u^{\prime} T^{\prime}$ values in the REVs. The change of $\left(u^{\prime} T^{\prime}\right)^{*}$ in the REVs with pore to throat size ratios of $1.63,3.04$ and 7.46 and for $\operatorname{Re}=1$ and 100 when $\varepsilon=0.7$ are shown in Fig. 7. By considering the legend of $\operatorname{Re}=1$, it can be easily seen that the value of $\left(u^{\prime} T^{\prime}\right)^{*}$ is small for $\beta=1.63$ and 3.04 since the most of area is
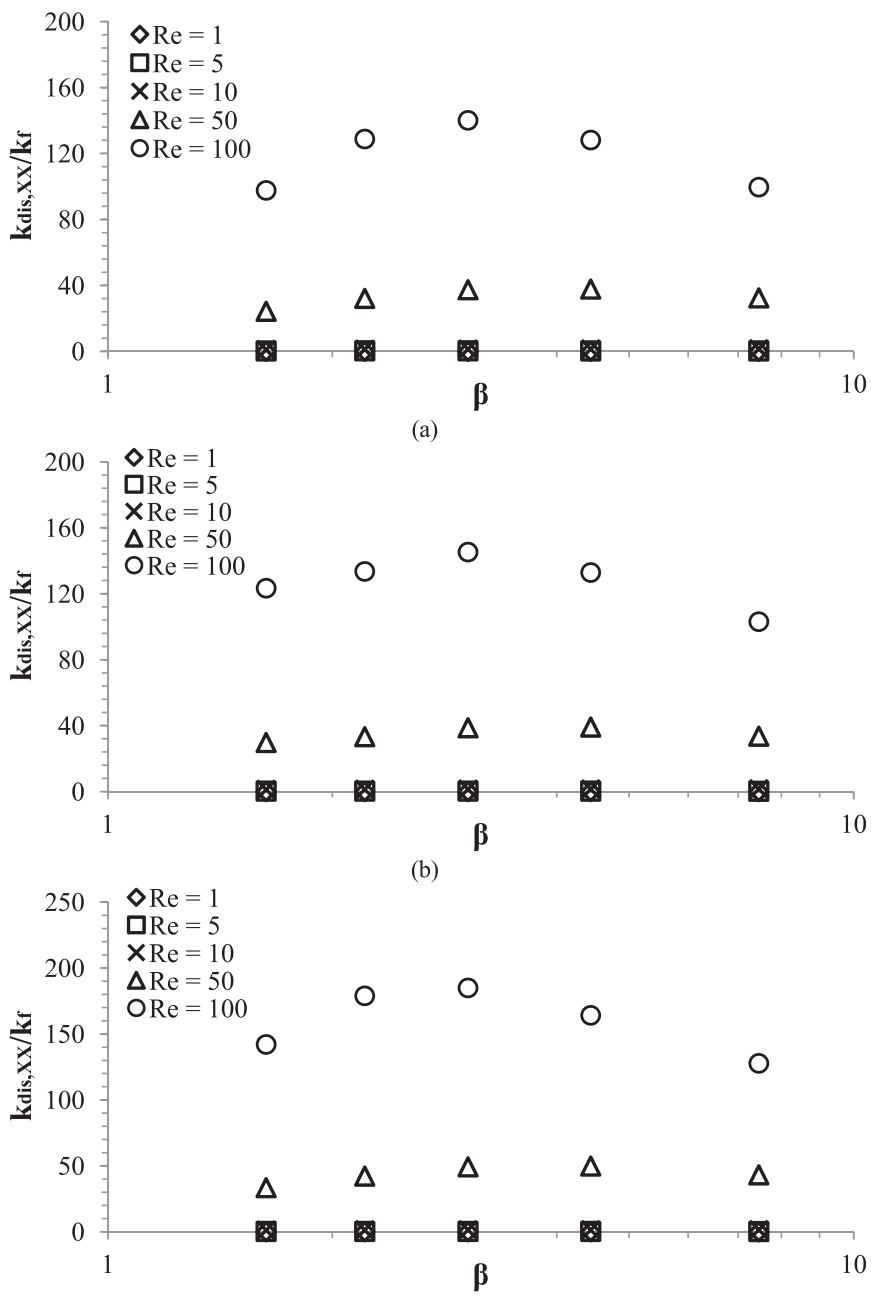

(c)

Fig. 8. The variation of the longitudinal thermal dispersion with $\beta$ for different Re for a) $\varepsilon=0.7$, b) $\varepsilon=0.8$, c) $\varepsilon=0.9$. 


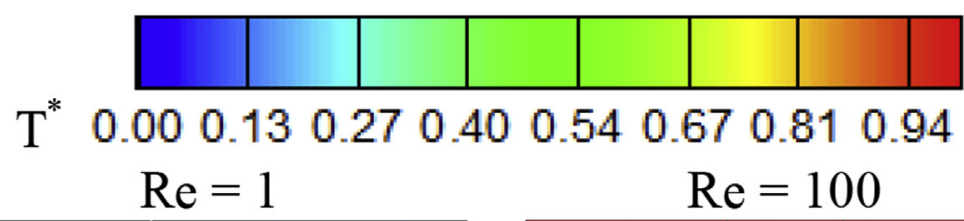

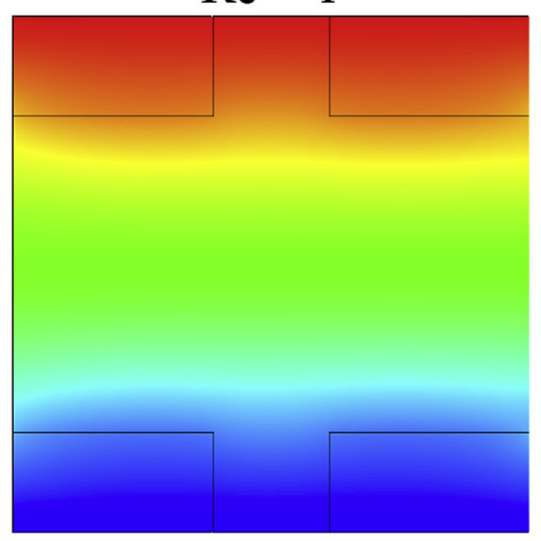

(a)

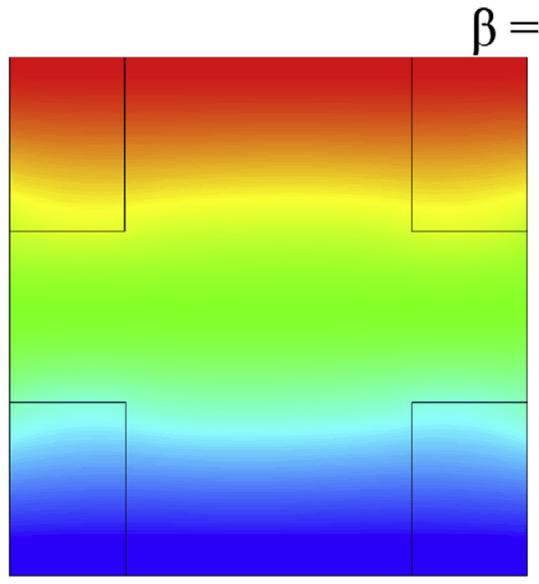

(c)

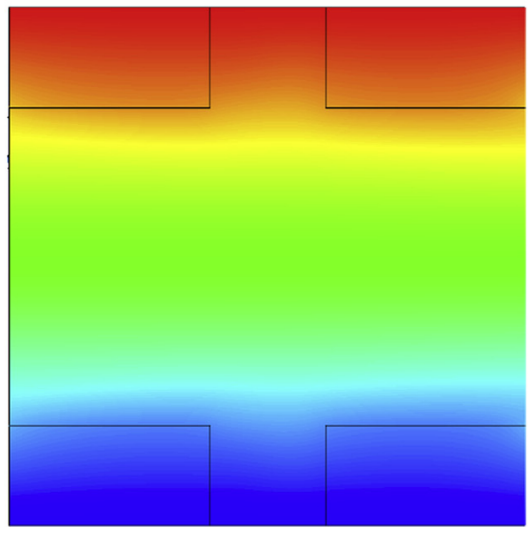

(b)

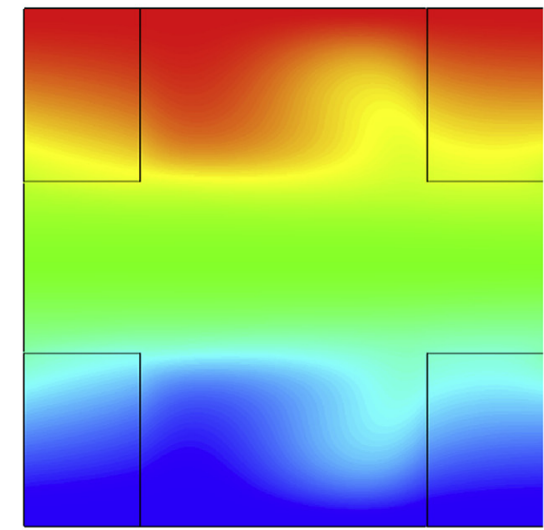

(d)

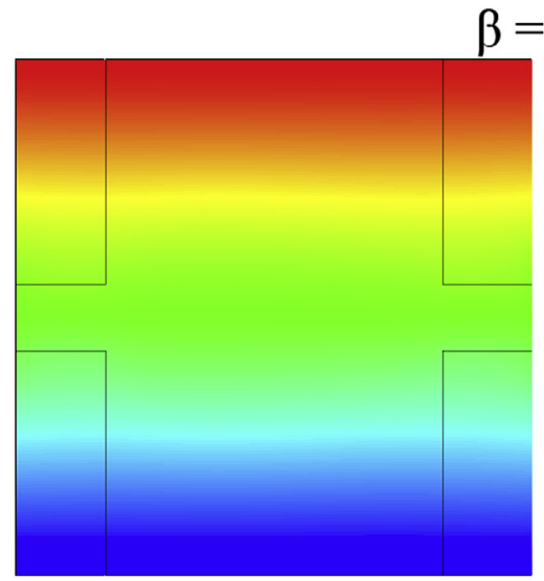

(e)

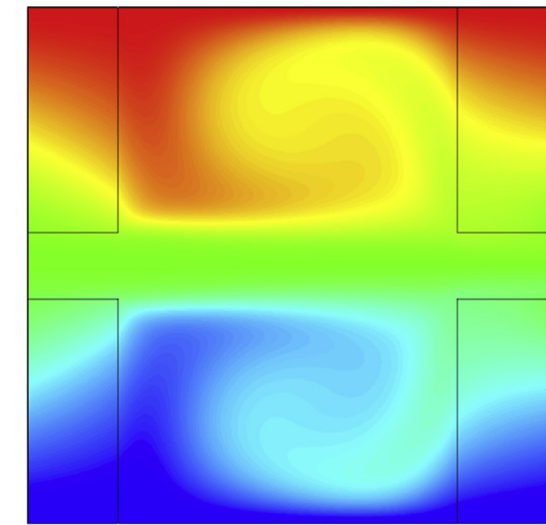

(f)

$$
\beta=7.46
$$

Fig. 9. The dimensional temperature contours in porous media with $\varepsilon=0.7$ when transverse temperature gradient exists. 
green (in the web version) representing the small values of $\left(u^{\prime} T^{\prime}\right)^{*}$. As it was mentioned before, the change of temperature can be seen only in $\mathrm{x}$ direction and the magnitude of $u-\langle u\rangle$ is very small since the velocities are small. That's why thermal dispersion is too low. For $\beta=7.46$, a high distribution value of $\left(u^{\prime} T^{\prime}\right)^{*}$, which is negative, exits at the inlet however it compensates with positive values of $\left(u^{\prime} T^{\prime}\right)^{*}$ at the outlet. That's why the rate of $\left(u^{\prime} T^{\prime}\right)^{*}$ even for $\beta=7.46$ is very small. If the legend of the $\mathrm{Re}=100$ in Fig. 7 is considered, it can be seen that the values of fluctuations considerably increase. In Fig. 7(b) the values of $\left(u^{\prime} T^{\prime}\right)^{*}$ in almost entire fluid region are very small, but the values are considerably greater than the ones in Fig. 7(a) in which $R e=1$. By increasing of pore to throat size ratio to 3.04, the green area (in the web version) in the middle region of fluid considerably enlarges which causes the increase of $\left(u^{\prime} T^{\prime}\right)^{*}$ value in total. In Fig. 7(f), a proper mixing of fluid (except in the narrow centre of fluid) can be seen. Heat transfer exchange between solid and fluid phases is high. The value of $\left(u^{\prime} T^{\prime}\right)^{*}$ in the solid decreases when it is compared with Fig. 7(d). That's why a decrease of thermal dispersion in $\mathrm{x}$ direction is expected.

The change of the ratio of the longitudinal thermal dispersion coefficient to the fluid thermal conductivity with the pore to throat size ratio for different Reynolds numbers and for porosities of 0.7, 0.8 and 0.9 are shown in Fig. 8. As expected, the longitudinal thermal dispersion coefficient is very small for $\operatorname{Re} \leq 10$, hence the thermal dispersion is negligible for the flows with $\operatorname{Re} \leq 10$. For higher Re, the dispersion effect along the longitudinal direction becomes stronger due to the increase of the convective heat transfer. It is observed that for the considered porosities in this study, the longitudinal thermal dispersion coefficient increases with pore to throat size ratio for the range of $1.63 \leq \beta \leq 3.04$ and then further increase of $\beta$ reduces $k_{\mathrm{dis}, \mathrm{Xx}}$. By the increase of $\beta$ from 1.63 to 3.04 , the longitudinal thermal dispersion increases almost $43 \%$, however, by further increasing of $\beta$ to 7.46 the thermal dispersion decreases $29 \%$. The maximum value of the longitudinal thermal dispersion is observed for the REVs with $\beta=3.04$. Hence, there is an optimum value of pore to throat size ratio for which the longitudinal thermal dispersion becomes maximum. The largest or narrowest throats have the lowest values of the longitudinal thermal dispersion coefficient. Fig. 8 also shows that, the value of the longitudinal thermal dispersion increases with the increase of porosity.

In order to determine thermal dispersion in the transverse direction, the macroscopic temperature gradient is applied in ydirection. The obtained dimensionless temperature contours for the REVs with porosity of $0.7, \beta=1.63,3.04$ and 7.46 , and for $\operatorname{Re}=1$ and 100 are presented in Fig. 9. The same flow conditions are employed for the computation of the transverse thermal dispersion coefficient; hence, the streamlines for these flows are the same with the ones that shown in Fig. 6. For the flows with low Reynolds number (Fig. 9(a), (c) and (e)) the macroscopically linear temperature gradient is not much disturbed by the flow since the convection effect in transverse direction is weak. For $\mathrm{Re}=100$ and pore to throat size ratio of 1.63 (Fig. 9(b)) the temperature distribution in the REV is similar to the REVs with $R e=1$. The areas of the gaps between particles in the upper and lower parts are small and there is no strong flow in the transverse direction. That's why; in spite of high Reynolds number, a linear temperature gradient in $\mathrm{y}$ direction is observed. For $\operatorname{Re}=100$, as the pore to throat size ratio increases, the main flow collides the walls of the solid particles at the right side of the REV and enters to the upper and lower gaps. Because of the mixing of main and secondary flows, the temperature distribution in the upper and lower gaps changes (Fig. 9(d)) and the linear temperature in y direction is disturbed. That's why the exception of the increase of thermal dispersion exists.
The variation of ratio of transverse thermal dispersion coefficient to the thermal conductivity of fluid with Reynolds number is shown in Fig. 10 for different values of pore to throat size ratio and porosities of 0.7, 0.8 and 0.9 . The transverse thermal dispersion is computed by using Eq. (19) for the REVs in which a temperature gradient in the transverse direction is created. If Figs. 8 and 10 are compared, it is observed that the transverse thermal dispersion coefficient values are much smaller than the longitudinal thermal dispersion coefficients for the inline arrangement of rectangular rods. As expected, the transverse thermal dispersion increases with the increase of Reynolds number, however the rate of increase depends on the pore to throat size ratio. For low pore to throat size ratios (i.e., $\beta=1.63$ ), the change of transverse thermal dispersion coefficient with Re is negligible. For high values of $\beta$, the transverse thermal dispersion considerably changes with Re. For instance, for $\operatorname{Re}=100$, the increase of $\beta$ from 1.63 to 3.04 causes the enhancement of the thermal dispersion by approximately $34 \%$. Further increasing of $\beta$ to 7.46 increases the thermal dispersion value by $114 \%$.

Most of the correlations suggested for the determination of thermal dispersion coefficient are expressed in terms of Reynolds and Prandtl numbers, porosity and solid-to-fluid thermal conductivity ratio as discussed in Ref. [3]. The present study shows that the thermal dispersion of two porous media having the same porosity,

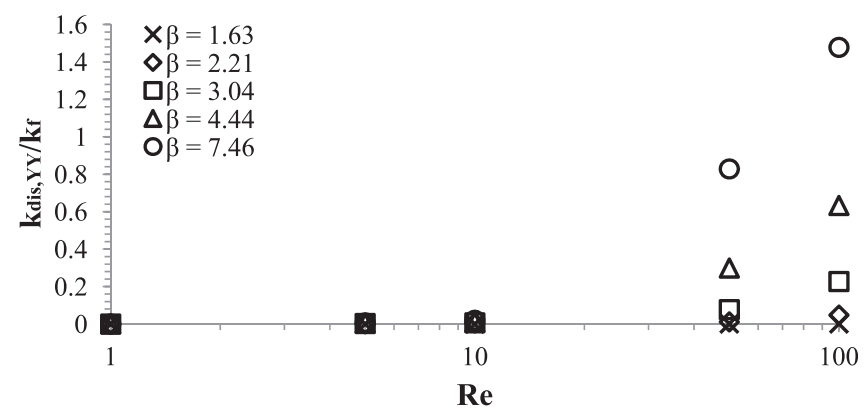

(a)
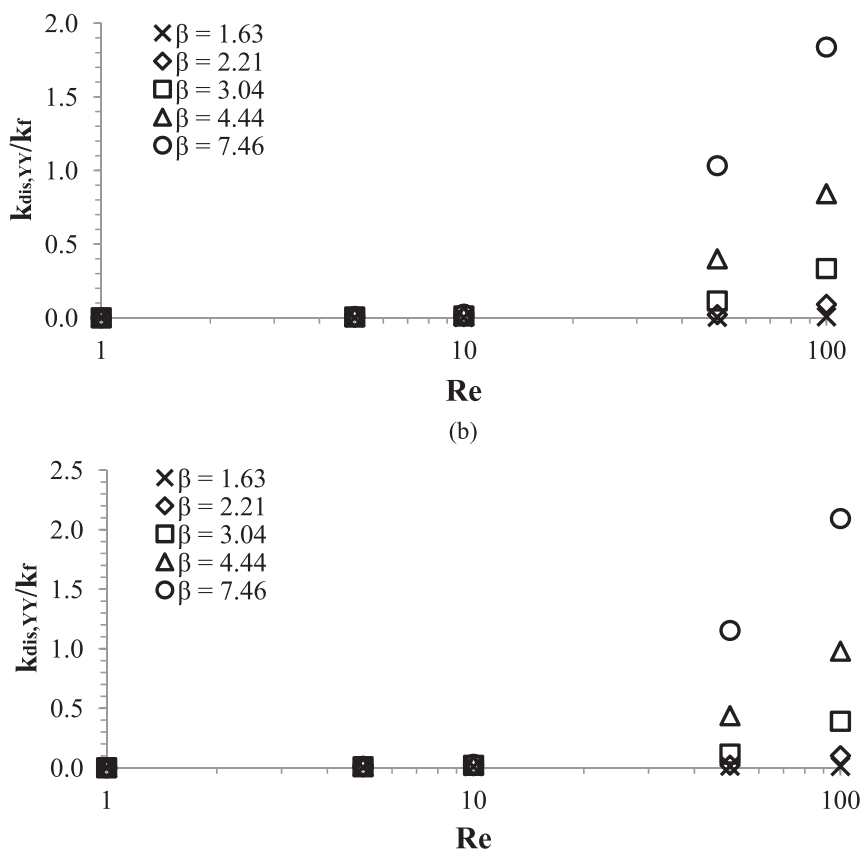

(c)

Fig. 10. The variation of the transverse thermal dispersion with Re and $\beta$ for a) $\varepsilon=0.7$, b) $\varepsilon=0.8$, c) $\varepsilon=0.9$. 
hydraulic diameter (or equivalent particle diameter) for the identical working fluid and flow conditions may be considerably different. As can be seen from Table 1, two porous media with different pore to throat size ratios ( $\beta=1.63$ and 4.44 ) have the same pore hydraulic and equivalent particle diameters and the same porosity. The thermal dispersion coefficients of these porous media are considerably different. For $\operatorname{Re}=100$ (and $\varepsilon=0.7$ ), the longitudinal and transverse values of $\mathrm{k}_{\mathrm{dis}} / \mathrm{k}_{\mathrm{f}}$ of the porous medium with $\beta=1.63$ are 97.62 and $1.22 \cdot 10^{-3}$, respectively, while for the porous medium with $\beta=4.44$, these values are 128.15 and 0.633 . Hence, the pore to throat size ratio plays an important role on the mechanism of thermal dispersion and it should be considered in the suggested correlations in order to prevent difficulties of correlations and achieve a relationship that is valid for various porous media.

\section{Conclusion}

The effects of pore to throat size ratio on the thermal dispersion coefficients in the longitudinal and transverse directions are investigated for the porous media consisting of long rectangular rods in inline arrangement, numerically. The following remarks can be concluded for the studied porous media in which the particles have inline arrangement:

- The longitudinal thermal dispersion coefficient is much higher than the transverse thermal dispersion coefficient.

- Both the longitudinal and transverse thermal dispersion coefficients are ignorable for low values of Reynolds numbers ( $\operatorname{Re} \leq 10$ ); however, for flows with $\operatorname{Re}>10$, as the convective heat transfer becomes stronger and the thermal dispersion coefficient increases with Re.

- The collision of the fluid particles to the walls of solid particles at the outlet of the REVs and the entrance of fluid into the gaps increases longitudinal thermal dispersion for the pore to throat size ratios between 1.63 and 3.04. However, further penetration of the fluid into the gaps and the increase of mixing effect reduce the longitudinal thermal dispersion. Hence, there is an optimum value of pore to throat size ratio for maximum longitudinal thermal dispersion in the longitudinal direction.

- The transverse thermal dispersion coefficient increases with Reynolds number, however the rate of increase depends on the pore to throat size ratio. For low values of pore to throat size ratio, the change of transverse thermal dispersion with $\mathrm{Re}$ is negligible.

- Both the longitudinal and transverse thermal dispersion coefficients increase with increase of porosity for the studied range of geometrical parameters.

- The value of thermal dispersion considerably changes with the pore to throat size ratio of porous media, hence in addition to porosity, solid to fluid thermal conductivity ratio, shape and arrangement of particles, Reynolds and Prandtl numbers, the pore to throat size ratio should be taken into account to predict the value of thermal dispersion for a porous medium.

The study should be expanded to different particle arrangements and pore configurations to provide a general idea on the effect of the pore to throat size ratio on the thermal dispersion. Further studies should be performed for higher thermal conductivity ratios and additionally the effect of thermal conductivity ratio on the thermal dispersion coefficients should be investigated.

\section{Acknowledgement}

The authors acknowledge the Scientific and Technological Research Council of Turkey, TUBITAK for 2211 scholarship for PhD students. This study was also supported by Izmir Institute of Technology with Scientific Research Project number of 2012-IYTE-02.

\section{References}

[1] S. Whitaker, The Method of Volume Averaging, Kluwer Academic, Dordrecht, The Netherlands, 1999.

[2] M. Kaviany, Principles of Heat Transfer in Porous Media, second ed., SpringerVerlag, New York, 1995.

[3] T. Özgümüş, M. Mobedi, Ü. Özkol, A. Nakayama, Thermal dispersion in porous media - a review on the experimental studies for packed beds, Appl. Mech. Rev. 65 (2013) 031001.

[4] M. Sahraoui, M. Kaviany, Slip and no-slip temperature boundary conditions at the interface of porous, plain media: convection, Int. J. Heat Mass Transf. 37 (6) (1994) 1029-1044.

[5] F. Kuwahara, A. Nakayama, H. Koyama, A numerical study of thermal dispersion in porous media, J. Heat Transf. 118 (1996) 756-761.

[6] F. Kuwahara, A. Nakayama, Numerical determination of thermal dispersion coefficients using a periodic porous structure, J. Heat Transf. 121 (1999) $160-163$.

[7] M.A. Saada, S. Chick, A. Campo, Analysis of hydrodynamic and thermal dispersion in porous media by means of a local approach, Heat Mass Transf. 42 (2006) 995-1006.

[8] M.H.J. Pedras, M.J.S. de Lemos, Thermal dispersion in porous media as a function of the solid-fluid conductivity ratio, Int. J. Heat Mass Transf. 51 (2008) 5359-5367.

[9] J. Xu, T.J. Lu, H.P. Hodson, N.A. Fleck, Analysis of thermal dispersion in an array of parallel plates with fully-developed laminar flow, Int. J. Heat Fluid Flow 31 (2010) 57-69.

[10] A.A. Alshare, P. Strykowski, T.W. Simon, Modeling of unsteady and steady fluid flow, heat transfer and dispersion in porous media using unit cell scale, Int. J. Heat Mass Transf. 53 (2010) 2294-2310.

[11] N. Jeong, D.H. Choi, Estimation of the thermal dispersion in a porous medium of complex structures using a lattice Boltzmann method, Int. J. Heat Mass Transf. 54 (2011) 4389-4399.

[12] http://machinedesign.com/materials/additive-manufacturing-comes-metalfoam.

[13] T. Ozgumus, M. Mobedi, U. Ozkol, Determination of Kozeny constant based on porosity and pore to throat size ratio in porous medium with rectangular rods, Eng. Appl. Comput. Fluid Mech. 8 (2014) 308-318.

[14] A. Nakayama, PC-Aided Numerical Heat Transfer and Convective Flow, CRC Press, Boca Raton, FL, 1995.

[15] C. Yang, A. Nakayama, A synthesis of tortuosity and dispersion in effective thermal conductivity of porous media, Int. J. Heat Mass Transf. 53 (2010) 3222-3230. 